\title{
TEXTANALYSE ODER TEXTINTERPRETATION? ÜBERLEGUNGEN AUS TEXTLINGUISTISCHER SICHT
}

\begin{abstract}
Text analysis or text interpretation? Some considerations from the text-linguistic viewpoint
Dealing with texts may mean using various research methods. The present paper discusses two such methodological approaches: text analysis and text interpretation. It is concerned, initially theoretically, with the question how these approaches can be explained from the text-linguistics point of view and separated from each other. After that a short text material is used to illustrate the thesis that analyzing and interpreting texts are two different forms of dealing with texts.
\end{abstract}

KEYwORDs: text linguistics, text analysis, text interpretation

\section{Einleitung}

Unter Sich-Beschäftigen mit Texten können im wissenschaftlichen Sinn verschiedenartige Arbeitsweisen verstanden werden. Ich möchte meine Diskussion auf die folgenden Herangehensweisen an Texte beschränken - die Textanalyse und die Textinterpretation - sowie darauf eingehen, wie ein bestimmter Umgang mit Texten textlinguistisch erklärt und - wie ich annehme - voneinander abgegrenzt werden kann. Wie man Texte $\mathrm{zu}$ analysieren und zu interpretieren hat, war Thema vorwissenschaftlicher und ist Thema wissenschaftlich fundierter Beschäftigung mit Texten, seitdem es Schrifttexte gibt. Denn Texte sind Ausgangs- und Zielpunkt rhetorischer, theologischer, stilistischer, literaturwissenschaftlicher, sprachgeschichtlicher, literaturgeschichtlicher, kulturanthropologischer und linguistischer bzw. sprachwissenschaftlicher Untersuchungen, schließlich auch textlinguistischer Untersuchungen (seit der Konstitution der Textlinguistik als Disziplin) und demnach auch textlinguistischer Untersuchungen mit soziologischen Aspekten (wie z. B. in der Diskursanalyse und Gesprächsanalyse). Diese Aufzählung der Disziplinen, die sich in ihrer jeweiligen zentralen Fragestellung mit Texten beschäftigen, ist natürlich nicht vollständig, kann aber als ein Warnen vor der m. E. allzu einfachen Neigung moderner sprachwissenschaftlicher Einzeltextuntersuchungen dazu betrachtet

Edyta Błachut - Uniwersytet Wrocławski, Wrocław, edyta.blachut@uwr.edu.pl 
werden, alles als textlinguistisch zu bezeichnen, nur weil es sich in der Untersuchung um Texte handelt. Die Beschäftigung mit der Einheit Text synonym als Analysieren und als Interpretieren zu bezeichnen, ist - wie noch zu diskutieren sein wird - nicht immer korrekt. Die bekannten Gebiete der Textinterpretation bzw. der Textauslegung dürften in erster Linie z. B. die Theologie und die Stilistik sein. Trotz der Übernahme vieler dieser Traditionen in die moderne Textlinguistik sind weder die Qualität des Textstils, noch die Beschaffenheit der sprachlichen Ausdrucksform, noch die ausgelegte Textbedeutung die grundlegenden Aspekte, die die Disziplin Textlinguistik kennzeichnen. Der Grundsatz der Textlinguistik was überhaupt Texte zu Texten macht, ist der Aspekt, der einer Analyse, und nicht der Interpretation unterliegt. Mein Ziel ist es, zunächst theoretisch (Kap. 2 und 3) und danach anhand von Textmaterial (Kap. 4) zu begründen, dass und wie sich Text-Analysieren und Text-Interpretieren voneinander unterscheiden.

\section{Was versteht man unter der Analyse eines Textes?}

Die in der Überschrift gestellte Frage ist in erster Linie durch die heute klar definierten Hauptaufgaben der Textlinguistik zu beantworten. Einer Textanalyse, die die sprachwissenschaftliche Disziplin der Textlinguistik zum Gegenstand hat, geht es um eine Klärung der folgenden Fragen: Was macht Texte zu Texten? Und was sind die typischen Merkmale, die vorhanden sein müssen, um von einem Text sprechen zu können? Die Aufgabenstellung der Textlinguistik und die theoretisch-begriffliche Grundlage wie Methoden der linguistischen Textanalyse sind bereits Gegenstand zahlreicher linguistischer Publikationen ${ }^{1}$. Dahinter verbergen sich jedoch keine einheitlichen Konzeptionen; gemeinsam ist den Publikationen aber, dass die oberste Bezugseinheit für die textlinguistische Analyse der Text ist, und nicht der Satz². Welche Position das Bild in der Textlinguistik einnimmt, hat inzwischen stark evolviert. Bilder konnten und können natürlich Bestandteile eines Textes sein, eines Textes, der durch die Textlinguistik als Mischtext bestimmt wird, vgl. z. B. so genannte Mischtexte mit der Dominanz von sprachlichen oder außersprachlichen Elementen wie Comics oder Plakate (z. B. Hausenblas, 1977). Heutzutage sind Bilder in manchen Texten (z. B. in Printmedien) aber so präsent wie nie zuvor, so dass eine linguistische Bildanalyse - mag sie überhaupt möglich sein - sogar im Begriff Bildlinguistik geprägt (Diekmannshenke \& Klemm

${ }^{1}$ Z.B. (hier nur eine knappe Auswahl aus der deutschsprachigen textlinguistischen Literatur) de Beaugrande \& Dressler (1981), Heinemann (1982), Heinemann \& Viehweger (1991), Canisius \& Knipf (1996), Vater (2001), Adamzik (2004), Brinker (2007).

${ }^{2}$ Erläuterungsweise muss hinzugefügt werden, dass in der textwissenschaftlichen Literatur heute allgemein angenommen wird, dass der Text, der als eine Satzfolge definiert wird, auch aus einem Satz bestehen kann. In diesem Fall unterliegt natürlich auch ein Satz einer textlinguistischen Analyse. Das Interesse am Satz bleibt auch in Form der Textsyntax, einem Teilbereich der Textlinguistik, als Zusammenbindung von Sätzen zu größeren Einheiten durch textverweisende Referenzmittel und Konnektoren bestehen. 
\& Stöckl, 2011) und aufgegriffen (von Große, 2011 u. a.) wird. Text/Textmodelle und Bild sind m. E. bei der linguistischen Analyse voneinander zu trennen, wenn auch nicht völlig isoliert voneinander zu untersuchen. Das Bild als Bestandteil eines Textes (wie z. B. in Printmedien) kann immer sein zusätzliches (z. B. durch Emotionen gesteuertes oder auf ästhetische Wirkung abzielendes) Begleitelement sein und durch seine Relation zum Text mit Bezug auf konkrete Analysebeispiele und die Kommunikationssituation zum Botschaftsträger werden. Was aber das Bild ,aussagt“", kann faktisch nur als Resultat einer unendlichen Menge potentieller und realer (individueller) Wahrnehmungsweisen aufgefasst werden. Bei der Bild-Produktion werden keine regulären, vom sprachlichen Handlungssystem bestimmten Beziehungen aktiviert, sondern die mentalen und psychischen Abläufe, die bei der Rezeption eines Bildes durch verschiedene Rezipienten nicht unbedingt gleich sein müssen. So hätten wir es bei Bildern mit einer Interpretation, nicht mit einer Analyse zu tun. Mit der Einbettung des Bildes in einen Textbildungsprozess kann sich eher die Psycholinguistik als eine Grenzdisziplin befassen, die Beurteilung des Bildes selbst verbleibt im Kompetenzbereich der Kunstwissenschaften, die Einbeziehung der Bilder in Werbeanzeigen ist - das stimmt wohl mit alltäglichen Erfahrungen überein - eine durchaus übliche Werbetaktik und steht als solche eher im Mittelpunkt des Interesses z. B. der Medienwissenschaften. Ob wir eine sich als Teildisziplin der Textlinguistik bzw. der Diskurslinguistik formierende Disziplin, die sich mit Bildern unter, über, neben und in Texten beschäftigt, benötigen, soll hier offen bleiben. Hier ist Folgendes wichtig:

Ich strebe eine analytische Trennung an, die der Wissenschaftler vornimmt, um die Einheit Text vor dem Hintergrund der eigentlichen Textlinguistik genau beschreiben zu können, bevor er eine individuelle Verarbeitung des Textes in seiner situativen und kontextuellen Gegebenheitsweise - aus seiner aktiven Interpretationsleistung heraus begründet vollbringt. Beim eigentlichen textlinguistischen Herangehen an den Text können zwei Blickrichtungen verfolgt werden: vom Satz zum Text als Ganzem oder vom Text zum Satz. Verfolgt man die Analyse vom Satz zum Text, so müssen auf (text-)grammatischer und (text-)syntaktischer Ebene verschiedene Referenzmittel und Konnektoren untersucht werden, die die einzelnen Sätze zu einem Text verbinden. Auf (text-)semantischer Ebene werden die einzelnen Sätze des Textes durch den Kontext und die gemeinsame Situation zusammengehalten; es muss untersucht werden, ob die Sätze eines Textes inhaltlich konnex sind (in der Textlinguistik als Herstellung der Textkohärenz bezeichnet, vgl. z. B. de Beaugrande \& Dressler, 1981). Verfolgt man die Analyse vom Text zum Satz, so stehen weitere Kriterien der Texthaftigkeit ${ }^{3}$ (z. B. Intertextualität) im Mittelpunkt der Analyse. Der Ausgangspunkt ist der Text als Satzfolge und als eigenständige Größe, wovon man zu den Bausteinen des Textes gelangt. Diese Bausteine können an der Textoberfläche auch syntaktisch-morphologisch, lexikalisch und semantisch analysiert werden.

\footnotetext{
${ }^{3}$ Texthaftigkeit, Textualität oder Textlichkeit, worunter man die Gesamtheit aller Eigenschaften versteht, die einen Text zum Text machen (im Sinn von de Beaugrande \& Dressler, 1981).
} 
Die pragmatische Ebene ist auch eine, auf der der Text theoretisch als Untersuchungsgegenstand betrachtet werden kann. Diese Ebene bietet aber einen völlig anderen Zugang zu Texten und zum Umgang mit Texten gegenüber dem mich interessierenden Sich-Beschäftigen mit Texten als die syntaktische und semantische Ebene. Man muss im Auge behalten, dass Texte grundsätzlich nicht im leeren Raum entstehen, sondern in einer konkreten kommunikativen Umgebung. Es gibt eine Reihe außersprachlicher Anhaltspunkte wie die Situation, den sozialen oder kulturellen Kontext, den Stil und die Intention des Autors des Textes, und andererseits den Grad des Verständnisses des Textes durch den Leser/Hörer, seine Erwartungen an den Text in Bezug auf Auffälligkeit, Originalität und Informativität etc. All diese Faktoren tragen aber in großem Maße zur individuellen Interpretation des Textes bei und rücken notwendigerweise das Interpretieren (nicht mehr das Analysieren) als eigenständigen und aktiven Umgang mit Texten ins Blickfeld. Man muss berücksichtigen, dass sich diese Faktoren wechselseitig ergänzen und - von der textlinguistischen Perspektive her - zu den sog. Textualitätsmerkmalen der Intentionalität und Akzeptabilität (i. S. v. de Beaugrande \& Dressler, 1981, S. 8-9) steigern, die ich hier mit Vater (2001, S. 42-44) eher als ,verwender- [und nicht text-] zentrierte“ Kriterien betrachten will, und zwar aus folgendem Grund: Intentionalität und Akzeptabilität sind eine allgemeine Voraussetzung für jede Art sprachlicher wie nichtsprachlicher Kommunikation, und kein (notwendiges) Kriterium für Textualität als ein Kriterium, das einen Text überhaupt zum Text machen würde (vgl. Vater, 2001, S. 42). Ich will Intention sowie Akzeptanz (in ihrer Wechselhaftigkeit), von der Seite eines Textrezipienten her gesehen, aus folgendem Grund als Interpretationskonstrukt(e) ansehen: Dem Textproduzenten kann eine bestimmte Intention nur unterstellt werden, und zwar gerade auf Grund einer aktiven Interpretationsleistung, die der Textrezipient durch eine eigene Verarbeitung des Textes vollbringt. Und man kann davon ausgehen, dass es dieselbe Intention ist, die der Textrezipient mit demselben Text in derselben Situation und demselben Kontext gehabt hätte, wenn er an Stelle des Textproduzenten stünde (gewünschter Effekt: Akzeptanz). Somit verlassen wir den Boden der Analyse des Textes, die sich m. E. a) zur Analyse der grundlegenden Kriterien des grammatischen und thematischen Textaufbaus (Textstruktur) eingrenzen lassen muss, b) Einsichten in die Regelhaftigkeit von Textbildung (bzw. Textaufbau, Textkonstitution) geben und c) die grundlegende Rolle des jeweiligen Textes/ der Textsorte im Kommunikationsprozess (Textfunktion bzw. Text-in-Funktion), d. i. den festgelegten Handlungscharakter eines Textes als die Art des kommunikativen Kontakts durch den Text bzw. mit dem Text (wie z. B. informieren oder appellieren), vermitteln soll.

\section{Was versteht man unter der Interpretation eines Textes?}

Im obigen Abschnitt wurde die folgende Unterscheidung der Beschäftigung mit einem Text angeboten: Das Analysieren eines Textes kann als sich nicht intuitiv vollziehender Vorgang mit einem objektiv feststellbaren Faktum als Analyseergebnis aufgefasst wer- 
den. Das Interpretieren eines Textes kann demgegenüber eine eigenständige und aktive Auseinandersetzung mit einem Text sein, die normalerweise individuell über eine aktive Hinzuziehung von eigenem Wissen ${ }^{4}$ bzw. über eine interpretative Fähigkeit beim Rezipienten erfolgt. In diesem Sinn bedarf das Interpretieren einer Präzisierung und Erklärung.

Zunächst sollen einige vorhandene Definitionen von Interpretieren in Bezug auf einen Text betrachtet werden ${ }^{5}$.

A) Der Duden. Deutsches Universalwörterbuch (2011, S. 775) sagt: Einen Text (z. B. ein Gedicht oder einen Gesetzestext) zu interpretieren bedeutet, den Text inhaltlich zu erklären, zu erläutern, zu deuten. Ich würde die folgenden Bezeichnungen aus dem Alltagsverständnis hinzufügen: den Text erhellen bzw. „dunkle“ Textstellen erklären und ihn bzw. sie (als Ziel) verständlich machen. In diesem Sinn ist Interpretieren als ein ,besser Sagen“ oder ein ,deutlicher Sagen“ dessen, was der Text dem Leser (oder dem Hörer) schon gesagt hat, zu verstehen, d. h. der zweite Schritt nach einem ersten, mehr oder weniger erfolgreichen Verstehen. Als Ergebnis oder Folge des Interpretierens ist normalerweise und erwartungsgemäß ein ,besser Verstehen“ des Textes anzusehen, wobei das Interpretieren und Verständlich machen des Textes nicht gleichzusetzen sind (dazu s. Pkt. C weiter unten). Das erwähnte „,besser/deutlicher Sagen“ kann sich nur sprachlich manifestieren, was dazu führt, das Interpretieren in Pkt. B) als eine sprachliche Handlung zu definieren. Also B): Dass Interpretieren von Texten eine eigene (sprachliche) Handlung ist, die ihrerseits neue Texte hervorbringen kann (,Texte über Texte“), darauf hat bereits Biere (1989, S. 25, zit. nach Busse, 1992, S. 187-188) hingewiesen:

Insofern als sich das Interpretieren auf sprachliche Gegenstände bezieht, auf Äußerungen oder Texte bzw. auf das Verständnis bestimmter Äußerungen oder Texte als parole-Ereignisse, ist es als sprachreflexiver oder metakommunikativer Akt zu verstehen, in dessen Vollzug selbst wieder sprachliche Zeichen hervorgebracht werden, die in einer zu spezifizierenden Weise auf vorgängige sprachliche Zeichen Bezug nehmen. Interpretationen sind in diesem Sinn eine bestimmte Art von ,Texten über Texte' [graph. Markierung im Original].

Aus dem in A) Gesagten (Interpretieren ist ein „besser/deutlicher Sagen“) und dem in B) Gesagten (Interpretieren von Texten ist sprachliche Handlung) ergibt sich C) Interpretieren (bzw. auch: Auslegen) von Texten als Anweisungen-Geben zum Verstehen. Interpretieren ist eine Sprachhandlung, die, wenn sie erfolgreich ist, dem Ziel dient, einen Text verständlich(er) zu machen. Ein „erfolgreich Sein“ der Interpre-

${ }^{4}$ Jede Interpretationshandlung muss immer als eine Explikation der eigenen Wissensbasis verstanden werden. Oder anders: Ein gegebener Wissensstand ist immer Voraussetzung und Ausgangspunkt des Interpretierens.

${ }^{5}$ Ich berücksichtige hier nicht das Interpretieren als Handlungsform, die die Deutung von jemandes Verhalten ist, und auch nicht die künstlerische Wiedergabe eines Musikstücks. Folglich ist es in Bezug auf den Text in (text-)linguistischer Hinsicht entscheidend, das Interpretieren auch nicht auf ein nachdenkliches Sich-Zurücklehnen gegenüber dem Text zu verkürzen, wie es wohl literaturwissenschaftliche Rezeptionstheorien auffassen würden. 
tationshandlung ist mit dem Wissensstand des Interpretierenden und der Adressaten verbunden (Biere, 1989, S. 248, zit. nach Busse, 1992, S. 245):

Genauso wie das Verstehen implizites Wissen voraussetzt, muß das Auslegen [synon. das Interpretieren, E. B.] einen Anknüpfungspunkt haben derart, daß es auch ein bestimmtes Wissen, ein (Teil-)Verständnis des auszulegenden Textes voraussetzen muß. In Art und Umfang dieses Vorwissens [...] ist letztlich Art und Umfang des verständlichmachenden Auslegens im praktischen Fall begründet.

Wenn der Interpretationsvorgang als eine Explikation der Wissensbasis des Interpretierenden und eine Aktivierung der Wissensbasis des Rezipienten aufzufassen ist, muss die Interpretationshandlung als ein „verständlich Machen“ nicht immer zu einem erfolgreichen Abschluss (zum Verstehen bzw. zu einem erweiterten, reicheren Verständnis) führen. Am Ende jeder Interpretation kann, wie auch Busse (1992, S. 190) hervorhebt, „,nicht eine allgemeingültige, für alle Situationen und alle möglichen und denkbaren Rezipienten verbindliche Auslegung stehen, sondern allenfalls ein Verstehen, das die jeweils »angesprochenen « Rezipienten befriedigt“. Damit ist gemeint, dass die Interpretationshandlung nie zu einer absoluten Klärung des Textinhalts führen kann, sondern nur zu einem relativen Abschluss der Interpretationshandlung, einer Anpassung an einen bestimmten Adressatenkreis. Hier kann man sich also fragen, inwieweit der traditionelle sowie der wissenschaftliche Terminus „Interpretieren“ in Bezug auf die linguistische Textuntersuchung anzuwenden ist.

Beide Umgangsweisen mit Texten, Analysieren und Interpretieren, lassen sich zwangsläufig, intuitiv wie reflexiv, voneinander abgrenzen. Das wesentliche textlinguistische Analyseinstrumentarium für die Textinterpretation liegt nicht so sehr im „verständlich Machen“ eines Textes, sondern darin, für ganz bestimmte Textsorten (sei es die mündliche Kommunikation im Alltagsleben, seien es Textsorten wie Gesetze, Predigt, Erzählung oder die wissenschaftliche Abhandlung) eine Konkretisierung derart vorzunehmen, dass die Texte die ihnen (institutionell) zugedachten Funktionen erfüllen können; damit kommen wir zu den Textualitätskriterien der Akzeptabilität, Intentionalität, Informativität und der Situationalität (i. S. v. de Beaugrande \& Dressler, 1981), bei denen wiederum das Risiko bestünde, das Ergebnis der Untersuchung sei kein objektives Faktum, sondern eine Frage der subjektiven Gewissheit der verstehenden Person, ihres intuitiven oder reflexiven Verstehens, ihrer Erwartungen an den Text (als Interpretationskonstrukt in obigem Sinn, vgl. Pkt. 1).

\section{Einzeluntersuchung eines Textes}

Ich nehme im Folgenden eine Zeitungsnotiz (aus: faz 9.06.2007, S. 2) als Beispiel. Es handelt sich hier um einen Text, in dem sich die sachbetonte mit der meinungsbetonten sprachlichen Darstellungsweise verbindet; das Thema ist in einen politisch-gesell- 
schaftlichen Zusammenhang einzuordnen. Alle graphischen Markierungen entstammen dem Original; die Nummerierung [1-8] von Abschnitten/Sätzen stammt von der Autorin:

Putins ,genialer Schachzug“" [1]

Die Moskauer Wirtschaftszeitung „RBK daily“ bezeichnet das Angebot Putins an die Vereinigten Staaten, gemeinsam eine Radarstation in Aserbaidschan zu betreiben, als genial [2]:

„Für die Idee spricht, dass ein Raketenschild im Kaukasus ganz Europa ohne Ausnahme schützen würde und nicht nur einen Teil wie bei der Variante mit Polen und Tschechien [3]. Das dürfte vor allem Litauen, Lettland und Bulgarien zufriedenstellen, die ja bereits die Amerikaner gebeten hatten, ebenfalls mit unter den Schutz kriechen zu dürfen [4]. Putin hat versprochen, von der Neuausrichtung der russischen Raketen auf Europa Abstand zu nehmen, sollte sein Vorschlag verwirklicht werden [5]. Putin hat mit Aserbaidschan einen genialen Schachzug unternommen [6]. Er kam als der große Antiheld zur G 8, der einen Kalten Krieg androhte [7]. Doch nach den Verhandlungen mit Präsident Bush hat der Kremlchef die Lage um 180 Grad gedreht [8]. (faz 9.06.2007, S. 2)

\subsection{Eine Analyse des Textes (text-zentriert)}

Aus der Einsicht in die textlinguistisch feststellbaren Bedingungen und Regeln der Textkonstitution ergeben sich die folgenden Merkmale des zitierten Textes.

Der Text ist eine texthafte Satzfolge im textgrammatischen Sinn (die Kohäsion): Es geht in diesem Text nicht um eine lose (ungeordnete) bzw. spontane Verknüpfung von Sätzen an der Oberfläche. Als Kohäsionsmittel gelten z. B. das Demonstrativpronomen das (am Anfang des Satzes [4]), wodurch das Subjekt des Satzes [3] wieder aufgenommen wird und das Personalpronomen er im Satz [7], wodurch das Subjekt des Satzes [6] wieder aufgenommen wird. Diese Elemente (als Folgenennungen) setzen also bestimmte Informationen voraus; sie verweisen (in anaphorischer Funktion) auf frühere Textstellen, an denen diese Informationen jeweils gegeben worden sind; in diesem Sinn ist die Satzfolge des Textes geordnet. Mit anderen Worten: Textfolgenennungen leisten eine Substitution.

Eine sinnvolle Relation zwischen den Sätzen/den abstrakten Konzepten liegt vor (die Kohärenz): Alle Sätze des Textes haben inhaltlich miteinander zu tun. Der Leser kann den sinnvollen inhaltlichen Zusammenhang auch ohne grammatische Verknüpfungsmittel erschließen. Die Sätze [2, 3, 4, 5] besagen etwas, was der Leser zu dem im Titel Angedeuteten [1] braucht, um ein Bild von dem im Titel Stehenden zu bekommen. Aus den Sätzen $[6,7,8]$ kann der Leser die Informationen zusammentragen, die ihm helfen, das im Titel Gesagte abzurunden. In Satz [8] fungiert das Subjekt der Kremlchef einerseits als grammatisches Verknüpfungsmittel (an der Oberfläche ist es als (kon-) textuelles Synonym für er [7] und Putin [6] zu betrachten; die Elemente sind referenzidentisch), andererseits greift der Leser zu seinem außersprachlichen Wissen, das er zum Verstehen der Sätze [6 u. 8] benötigt. Die Textkohärenz kommt in den meisten 
Fällen erst nach dem verarbeiteten Textwissen und dem allgemeinen Weltwissen/der Aktivierung situativer Umstände zustande.

Der Text hat ein Thema, i. S. v. „,er sagt etwas über etwas aus“ (Textthema und Thema-Rhema-Struktur von Sätzen als Gerüst des Textaufbaus): Das Thema des Textes ist eine nichtsprachliche Größe, die durch den Text versprachlicht wird und in einen Wissenszusammenhang eingebettet ist (das Thema eines Textes bestimmt in diesem Sinn weitgehend die Kohärenz-Beziehungen im Text, s. oben). Oder umgekehrt: Der Text kann als entfaltetes Thema angesehen werden. Textlinguistisch betrachtet kann das Thema, muss aber nicht, im Text explizit genannt werden. In diesem Text stellt der Titel [1] das Thema des Textes explizit dar ${ }^{6}$. Die Thema-Rhema-Gliederung der Sätze des Textes berücksichtigt, dass nicht nur der Gesamttext, sondern jeder Teil des Textes bis hinunter zum Satz ein Thema hat, dass die Sätze normalerweise als Teile von Texten verwendet werden (vgl. z. B. Hellwig, 1984, s. auch zur Definition des Textes in Pkt. 2). Entscheidend ist für die Verhältnisse im Satz, woran ein jeweiliger Satz anschließt. Ein Versuch, den Text zu zerlegen, zeigt eine lineare thematische Progression, wonach das Rhema (in der traditionellen Auffassung: die neue Information innerhalb eines Satzes, vgl. Reis, 1977) des einen Satzes [2] zum Thema (die alte/bekannte Information, in der Auffassung von Reis, 1977) des folgenden Satzes [3] wird, usw. Thema und Rhema entwickeln sich im Text weiter. Das Thema in der Thema-Rhema-Analyse ist nicht als Thema i. S. v. Textinhalt zu verstehen.

Zur Wiederholung sei kurz zusammengefasst: Die wichtigsten Ordnungsprinzipien im Text, die dafür verantwortlich sind, wie ein Text zusammengehalten wird, wie er überhaupt zu einem Text wird, sind (a) ein Thema, zu dem etwas geäußert wird, und (b) entsprechend gewählte sprachliche und semantische Elemente (Konnektoren), die die spezielle Verbindung von einzelnen Strukturelementen des Textes vermögen. Den Terminus Konnektor verwende ich als Sammelbegriff für alle textkonstituierenden Mittel der Konnexion (auch: Mittel der Verbindung, Verknüpfung, Textverflechtung). Diese Mittel treten in Texten in verschiedensten Konstellationen zueinander auf. In diesem konkreten Text - einer Zeitungskurznachricht - liegen die folgenden Mittel vor.

- Im weitesten Sinn gehört zur Konnexion in diesem Text die thematische Progression sowie die Einbettung in einen konkreten situativen Kontext. Dass der Autor dieses Textes wohl annimmt, dass der Leser von dem berichteten Ereignis bereits etwas wissen kann, ist durch die explizite Einführung der Informationsquelle in [2] und durch die bestimmten Artikel ohne Vorerwähnung begründet. Das (Welt-)Wissen des Lesers, das die bestimmten Artikel voraussetzen, entstammt vermutlich nicht diesem konkreten Text, sondern z. B. (mehreren) früheren Texten, entsprechenden Meldungen derselben Zeitung in den vorausgegangenen Tagen, Fernseh- oder

${ }^{6}$ Text-Titel sind als explizite Mittel gerade in der Zeitung typisch; van Dijk (1980, S. 50) spricht hier vom „Themawort” oder „Themasatz”. 
Radionachrichten etc. Der Text zeigt in diesem Sinn einen makroanaphorischen Anschluss an frühere Texte.

- Als satzinterne Konnektoren treten Relativpronomina (wie in [4] u. [7]; die dadurch angeschlossenen Sätze haben geradezu die Funktion, den inhaltlichen Kern zusammenzufassen bzw. notwendige, neue Informationen einzuführen), Possessivum in [5], Kongruenz (in allen Sätzen) und syntaktische Elemente (Ergänzungen, Angaben, Attribute), die jeweils den Satz ,zusammenhalten“ (wie in [2] zu Angebot, in [4] zu bieten, in [5] zu versprechen) auf. Nun sollen die beiden Relativsätze in [4] und [7] noch etwas genauer betrachtet werden. Eine der satzorientierten Relativsatztypologien unterscheidet restriktive (notwendige, obligatorische) und nichtrestriktive (freie, fakultative) Relativsätze. Sind diese Sätze nun in ihrer semantischen Funktion restriktiv oder nichtrestriktiv? Sie sind sicher nicht restriktiv im traditionellen Sinne, in dem sie obligatorisch sind. Sie sind aber auch nicht fakultativ in dem Sinne, dass sie weggelassen werden können. Sie sind vor dem Hintergrund einer textlinguistischen Betrachtung insofern obligatorisch bzw. notwendig, als sie zum besseren Textverstehen nötige Informationen nachliefern und so, wo wie in [7] grammatisch möglich, den bestimmten Artikel legitimieren. Ohne den Relativsatz in [7] wäre der große Antiheld nämlich unverständlich. Diese Funktion der Relativsätze bezeichne ich als textgrammatische Funktion.

- Satzextern (über die Satzgrenze hinaus) wirken Verweispronomina und kontextuelle Synonyme (wie das in [4], er in [7], der Kremlchef in [8]; er und der Kremlchef sind referenzidentisch) sowie einfache Wörter und Wortgruppen, die wiederholt werden; auch bei ihnen wird eine vorhergehende Äußerung vorausgesetzt und sie sind in diesem Sinn satzverbindend und textkonnex (genialer Schachzug in [1] und [6], genial in [1], [2], [6]).

- Die spezielle Verbindung im Text (zwischen den Sätzen sowie längeren Textabschnitten) vermögen auch die Vereinigten Staaten in [2], die Amerikaner in [4] und Präsident Bush in [8] herzustellen, die zwar nicht koreferent sind, aber als stilistisches Umgehen der als unschön bewerteten Wiederholungen zu betrachten sind. Die Ausdrücke stehen in einer Kontiguitätsrelation zueinander. Die Folgeausdrücke bezeichnen immer etwas, was im weitesten Sinne ein Teil dessen ist, was der zuvor angeführte Ausdruck bezeichnet.

- Zur Wiedergabe räumlicher, zeitlicher und logischer Beziehungen dienen im Text Adverbien und Partikeln wie vor allem, ja, bereits, ebenfalls in [4], doch in [8]; ihre Aufgabe ist die Textorganisation in der Hinsicht, dass sie ohne vorausgegangene Äußerungen nicht eingesetzt werden können. Bei ihrem Fehlen aber würde der Text den Textcharakter verlieren und der Zusammenhalt ginge verloren.

- Als Pro-Wörter (zur Konnexion) dienen auch die Nomina wie Angebot in [2], Idee in [3], Vorschlag in [5]. Die Gliedstellung in [3] sorgt übrigens besonders dafür, dass die Folgenennung (für die Idee) möglichst nahe an ihrer Vorerwähnung (genial) steht, dass also die Verbindung der Sätze [2] und [3] möglichst eng ist (X-genial 
$\rightarrow$ für die [Genialität der] Idee spricht...). Ich bezeichne dies aber nur als eine Tendenz, nicht als Regel, mit der Voranstellung der Glieder, die kein Subjekt sind. - Die (globale) kausale und logische Beziehung im Text - als global verstehe ich die Beziehung zwischen der Überschrift und allen übrigen Textabschnitten bis zum letzten Satz - wird schließlich nicht immer und bei jedem Satz explizit ausgedrückt; oft ist sie nur aus dem Kontext erschließbar (u. a. dank Thema-Rhema-Ketten; vgl. das oben Gesagte zur Konnexion im weitesten Sinne). In diesem konkreten Text: Der Anfangssatz [2] und der Schlusssatz [8] rahmen gewissermaßen den Kern des Textes ein; sie haben hier eine deutlich textstrukturierende Funktion.

Die einschlägige Fachliteratur wird den Text ,Zeitungsnachricht“" zu den sachinformierenden Texten (vgl. Große, 1976) oder Informationstexten (Dimter, 1981; Brinker, 2007) zählen ${ }^{7}$. Dieser Zuordnung entspricht die Charakterisierung des Textes nach der dominanten kommunikativen Funktion Informieren: Inhalt an die Kommunikationsteilnehmer übermitteln. Der Text soll demnach in Zusammenhang damit betrachtet werden, wozu er dient. Eine textlinguistische Analyse sollte eigentlich eine Vermittlung zwischen den linguistischen Strukturmerkmalen des Textes und seinen pragmatischen Dimensionen sein. Für diesen konkreten Text gilt:

- Die Intention des Senders ist es, dem Leser etwas mitzuteilen, ihn über etwas zu informieren.

- Diese informative Funktion wird prototypisch durch explizit performative Verben wie informieren, mitteilen, berichten, benachrichtigen, melden etc. indiziert (vgl. Brinker, 2007, S. 113). In dem Text aber stellt der Sender den thematischen Sachverhalt als tatsächlich dar, indem er sich auf den Sicherheitsgrad dem Textinhalt gegenüber beruft, und zwar in Satz [2] durch $X$ bezeichnet $Y$ als $Z^{8}$. Die Sicherheit des Wissens wird durch den Sender in Satz [4] durch das Modalverb dürfte in seiner subjektiven Bedeutung, und in Satz [5] durch das Modalverb sollte im Kontext einer Bedingung eingeschränkt. Die Informationsfunktion verbindet sich mit der Bewertung des thematisierten Sachverhalts. Der Sender informiert über die Einstellung gegenüber dem Objekt der Bewertung: in Satz [1] und [6] durch das Adjektiv genial, in Satz [7] durch das Substantiv Antiheld, in Satz [8] durch den Ausdruck die Lage um 180 Grad drehen.

- Die Wahl der Tempora und Modi in diesem Text suggeriert aus textgrammatischer Sicht das Folgende: (a) (Erzähl-)Bericht, (b) in anderen Textteilen wird deutlich

${ }^{7}$ Als Basiskriterium zur Textklassifikation gilt die Textfunktion, was dadurch begründet ist, dass - nach Dimter (1981, S. 116, zit. nach Brinker, 2007, S. 145, auch von der neueren Fachliteratur akzeptiert) - über 80\% der untersuchten alltagssprachlichen Textsortenkonzepte gerade Informationen zu Textfunktionen enthalten.

${ }^{8}$ Explizierende Paraphrasen zur Verdeutlichung der Informationsfunktion, in Verbindung mit der Einstellung des Senders zum Sicherheitsgrad bzw. Wahrscheinlichkeitswert des übermittelten Sachverhalts werden in der Fachliteratur z. B. als Assertionssignale (Große, 1976, S. 76) oder Vermutungssignale (Große, 1976, S. 79) klassifiziert. 
darauf verwiesen, dass der Sender zitiert, (c) in anderen Textteilen wiederum abstrahiert der Autor davon, indem er seine Stellungnahme/Vermutung signalisiert.

- Die referierende Darstellungsweise kommt in dem Text darin zum Ausdruck, dass die Textteile in der grammatischen Form der indirekten Rede wiedergegeben werden: im Konjunktiv in Satz [3], durch Angabe der Informationsquellen in Verbindung mit dem entsprechenden Verb im Indikativ: X bezeichnet etwas als $Y$ in Satz [2] und $X$ hat versprochen in Satz [5]. Dies spricht für die erwartete „Neutralität“, charakteristisch für die Textsorte (Zeitungs-)Nachricht ${ }^{9}$.

- Die analysierte Zeitungsnachricht ist ein monologisch konstituierter Text.

- Die Wahl des Tempus (Präsens in den Sätzen [2] und [3], Futur in Satz [3], Plusquamperfekt in Satz [4], Perfekt in den Sätzen [5], [6] und [8] und Präteritum in Satz [7]) signalisiert textlinguistisch ${ }^{10}$ Folgendes: das Präsens als besprechendes Tempus (das Futur als Vorschautempus und das Perfekt als Rückschautempus) im Text geht es um eine Angelegenheit/einen Sachverhalt von aktueller Relevanz; das Präteritum als erzählendes Tempus (das Plusquamperfekt als Rückschautempus) - im Text geht es um eine Angelegenheit/einen Sachverhalt von weniger aktueller Relevanz/von keiner Relevanz. Vgl. z. B. den letzten Textabschnitt: Das Präteritum in Satz [7] verweist darauf, dass das Ereignis im Moment des Berichtens eine kleinere Rolle gegenüber dem in [8] Gesagten spielt, das zur Zeit der Nachrichtendarstellung noch aktuell ist. Tempora haben eine text(-sorten-)spezifische Funktion insofern, als sie dazu dienen, das Thema in einen größeren politisch-gesellschaftlichen Zusammenhang einzuordnen.

\subsection{Eine Interpretation des Textes (verwender-zentriert)}

Die Formulierung verwender-zentriert will ich auf Einstellungen, Gefühle, Meinungsänderung, Verhalten $u$. a. seitens des Textproduzenten und Textrezipienten beziehen. Hier können Faktoren angeführt werden, die durch das Vorkommen bestimmter sprachlicher Ausdrücke, darunter unter anderem rhetorischer Figuren, definiert werden. In manchen Text(-sort-)en kommen auch außersprachliche Elemente als Faktoren in Frage (u. a. in Texten mit Appellcharakter wie Werbungen, Kochrezepte etc.), denen Text- bzw. Text-Bild-Forscher die Eigenschaft zuschreiben, beim Rezipienten bestimmte Gefühle auszulösen. Es handelt sich dabei unter anderem um die Faktoren, welche

\footnotetext{
${ }^{9}$ Vgl. hier die Bemerkung von Brinker (2007, S. 116), dass das für Nachrichten verbindliche Prinzip der sachbetonten Darstellung nicht bedeuten muss, dass Nachrichtentexte völlig frei von Bewertungen sind. In diesem Zusammenhang lassen sich die Wertwörter im vorigen Punkt begründen. Die informative Textfunktion kann mit einer meinungsbetonten sprachlichen Darstellung kompatibel sein, ohne den Leser in seiner Haltung beeinflussen zu wollen.

${ }^{10}$ An dieser Stelle möchte ich auf eine kurze Diskussion über den textlinguistischen und texttypologischen Charakter von Tempora in Canisius \& Knipf (1996, S. 157-165) verweisen.
} 
die Situation der Textrezeption als Teil eines komplexeren Kommunikationsprozesses kennzeichnen. Dazu habe ich das Folgende anzumerken:

Was innerhalb einer allgemeinen Theorie der Kommunikation näher spezifiziert wird, ist die Position des Textproduzenten mit seinen Zielen und Fähigkeiten, Vorstellungen und Annahmen und die Rolle der Intentionen des Autors für das Sprachverstehen. Wenn man davon ausgeht, dass jede Kommunikation durch die Intention des Senders zustande kommt, so kann man auch formulieren, dass sie für die Textkonstitution kennzeichnend ist. De Beaugrande \& Dressler (1981, S. 8) machen gerade die Intentionalität zum Textualitäts-Merkmal. Canisius \& Knipf (1996, S. 23) begründen die Intentionalität auf textueller Ebene wie folgt:

Sie [die Absicht des Senders, E. B.] verkörpert das Kriterium, das den Text von einer losen Abfolge von Sätzen unterscheidet, insofern muß ihr mehr Beachtung geschenkt werden als einer einfachen kommunikativen Intentionalität. Sie ist quasi der erste Schritt für die Textkonstitution, durch die Intentionalität und die anderen unentbehrlichen Elemente kann aus Bausteinen erst ein Text gebildet werden.

Wie Intentionalität so sind auch Akzeptabilität und Informativität (auch von de Beaugrande \& Dressler, 1981 zu Textkriterien gemacht) wichtige Voraussetzungen jeder Kommunikation sowie jeder sprachlichen Äußerung und somit jedes Textes: Ein Textrezipient geht immer mit Erwartungen an einen Text heran. Er hat immer ein Informationsbedürfnis. Er bekommt durch den Text Informationen und Wissen, um den gesamten Text zu verstehen. Auf sprachlicher Ebene spielen z. B. der der Situation angepasste Stil und die Sprachvarianten eine Rolle, durch die der Textrezipient den Text rezipiert. Auf kommunikativer Ebene ist hier das gemeinsame Wissen der Kommunikationsbeteiligten eine wichtige Bedingung. Die Erwartungen des Rezipienten können aber auch Annahmen über die möglichen Intentionen des Textproduzenten enthalten. Ich will hier die Wörter „Annahme“ und „möglich“ betonen. Denken wir z. B. an rhetorische Figuren, Metaphern, Wortspiele in Nachrichten, Berichten und anderen Texten mit ihrer spezifischen sachbetonten Themaentfaltung oder an Bilder und andere außersprachliche Mittel, die Werbetexte begleiten oder ergänzen. Sicher manifestiert der Sender seine Intention durch diese Mittel. Man kann davon ausgehen, dass der Rezipient annehmen würde, dass der Autor mit dem geäußerten Wort, der Paraphrasierung, dem eingelegten Bild etc. dasselbe gemeint haben wird, was er selbst, hätte er diesen Text produziert, in dem spezifischen Kontext bzw. der ähnlichen Situation damit gemeint haben würde. So wird deutlich, dass die Darstellung im Text einen gewissen Interpretationsspielraum lässt. Oder umgekehrt, die Textrezeption ist gewissermaßen relativ: Der gleiche Text kann von mehreren Empfängern je nach ihrer (sprachlichen) Sensibilität und Kompetenz und auch nach ihrem individuellen Weltwissen unterschiedlich interpretiert werden. Falsch wäre die Behauptung, dass eine Identität des Wissens bei allen Beteiligten einer Sprachgemeinschaft bzw. bei allen Rezipienten eines Textes besteht. 
Die Interpretation unseres Textes kann durch folgende Fragen gesteuert werden: Welches Vorwissen hat der Rezipient und wie greift sein Vorwissen in das Verstehen dieses Textes ein? Welche sprachlichen Fähigkeiten hat er bzw. braucht er, um diesen Text zu verstehen?

- Das erfolgreiche Herangehen an diesen Text hängt in großem Maße von den politischen Vorkenntnissen des Lesers ab. Die Annahme des Textproduzenten zu möglichen Einordnungsversuchen des Rezipienten, was die Einbettung der mitgeteilten Informationen in einen konkreten politischen Kontext anbelangt, manifestiert sich in der Wahl der Vokabeln und Wortformen, die einen Fachbereich benennen, ohne sie erklären zu müssen (Radarstation, Raketenschild, Neuausrichtung der Raketen, $G 8)$. Der Rezipient ist aufgefordert, kontextuell angemessene Wortbedeutungen zu aktivieren, vgl. [...] unter den Schutz kriechen zu dürfen in Satz [4] oder Antiheld in Satz [7]. Die Frage nach der Erwartungshaltung des Textrezipienten gegenüber dem Text betrifft sein historisches Wissen (vgl. einen Kalten Krieg androhen in [7]) und seine Fähigkeit, den Hauptgedanken zu erfassen und auf die aktuelle politische Situation zu beziehen.

- In einen politischen Kontext sind andere, nicht politische Konzepte eingebaut, deren Verstehen auch eine wichtige Voraussetzung ist, um sich den gesamten Text zu Eigen zu machen. Es handelt sich um die Ausdrücke (genialer) Schachzug, den Schachzug unternehmen in [1] und [6] und die Lage um 180 Grad drehen in [8]. Beim letztgenannten Ausdruck geht es zudem um die dem Textrezipienten unterstellte Fähigkeit, Schlussfolgerungen zu ziehen (Endposition im Text).

- Die Ausdrücke den Schachzug unternehmen und die Lage um 180 Grad drehen sind metaphorisch und man kann annehmen, dass sie eine Stellungnahme des Autors enthalten und als Interpretationsanleitung dienen (können), indem sie dem Leser durch ihre feste Bedeutung seine eigene Interpretation der Fakten abnehmen. Ansonsten sind sie auch eine Wertungsvorausnahme. Schachzug bezeichnet außerhalb des Schachspielkontexts eine geschickte Handlung zur Erreichung eines bestimmten, dem eigenen Interesse dienenden Ziels; hier wird die Metapher durch das Adjektiv genial in der Bewertung des Sachverhalts verstärkt. Die Lage um 180 Grad drehen (zum entgegengesetzten Ausgangspunkt übergehen) präsupponiert, man sollte den Kremlchef wertschätzen, bewundern. Die Wahl der metaphorischen Ausdrücke kann man auch so interpretieren, dass der Sender versucht, sich dem politischen Problem über die Formulierungen der Alltagssprache zu nähern und auf diese Weise den Text (und den komplizierten politischen Sachverhalt) verständlicher zu machen. Aber auch sprachliches Wissen auf der Ebene der Metaphorik ist subjektiv und identische Vorgänge auf den Ebenen der Textproduktion und der Textrezeption sind eher der Idealfall.

- Die Metaphorik gehört zu den Faktoren, die einen Text für die Situation aktuell, interessant, auffallend und originell machen - so auch in diesem Text. Die vorgege- 
bene Textorganisation ist sicher gewollt: Schlagzeile (Putins ,, genialer Schachzug “), Fließtext (Putin hat... einen genialen Schachzug unternommen) und Schlusssatz (Doch nach den Verhandlungen ... hat der Kremlchef die Lage um 180 Grad gedreht). Für die Interpretation der Ausdrücke in diesen Positionen übernehme ich die Feststellung von Koller (1975): Die Schlagzeile steuert die Rezeption des Textes, und zwar auch in Hinblick auf die Bewertung des Thematisierten. Der Autor will, nach meiner Interpretation, die Aufmerksamkeit des Lesers auf das Wichtige lenken. Ein Fließtext ist der Ort, in dem die Ausdrücke (oder ihre Teile) häufig zum zweiten Mal erscheinen und als Bestätigung der vom Autor angenommenen Darstellungsrichtung angesehen werden können. Am Ende des Textes fassen sie die Auseinandersetzung/ den Inhalt zusammen, fordern zur Reflexion auf, bewerten häufig auch, und gerade an dieser Position lassen sie die Möglichkeit der Eigenbewertung seitens des Lesers zu. Der Autor nimmt an, dass der Leser dieser Textorganisation folgen kann.

- Der Autor geht im Weiteren auch davon aus, dass der Leser die grammatischen Strukturen erkennen kann, die eine bestimmte Rezeptionshaltung steuern: Der Rezipient soll unterschiedlich auf erzählende und besprechende Textabschnitte reagieren, direkte Textwiedergabe von distanziertem Textwiedergeben unterscheiden. In diesem Text kann z. B. die Setzung der Anführungszeichen der Interpretation unterliegen. In der Schlagzeile [1] hat man auf den ersten Blick den Eindruck, dass der Ausdruck von der genannten Person kommen kann (auch durch den Gebrauch des Genitivs). Der Textinhalt begründet einen anderen Fall, nämlich nur die Zuschreibung des Sachverhalts zu der Person. Dann bietet sich eine andere Interpretation an: Vielleicht wollte der Autor signalisieren, dass man den Ausdruck nicht ernst nehmen müsse, oder zumindest nicht wortwörtlich. Dass der Ausdruck schon ernst (im politischen Sinne), aber nicht wortwörtlich (im sprachlichen Sinne) zu interpretieren ist, wird aus dem Textinhalt klar. Wollte der Autor dann vielleicht die Aufmerksamkeit des Lesers auf das Wichtigste in der Nachricht lenken? Die Anführungszeichen in [2] sind relativ einfach zu verstehen: Sie signalisieren die Quellenangabe. Durch umrahmende Anführungszeichen (ab [3] bis [8]) wird, so kann man annehmen, der direkt zitierte Text kenntlich gemacht.

\section{Schlussbemerkung}

Im Interpretationsprozess des Textes, wie oben (in 4.2.) angeboten, kann davon ausgegangen werden, dass auf allen Ebenen der Sprache ,subjektives“ Textverstehen oder sogar Missverständnisse eines Textes möglich sind. Möglicherweise kann, beginnend bei den elementaren sprachlichen Fähigkeiten (wie z. B. dem Erkennen grammatischer Strukturen, Interpunktionszeichen und ihrer Funktion) über stilistische Sprachempfindlichkeit (wie dem Verstehen von Metaphern oder dem Folgen der Textorganisation), von Ebene zu Ebene eine Veränderung des Verstehens angenommen werden, von weitge- 
henden intuitiven Abläufen hin zu zunehmend bewussteren Vorgängen. Interpretation ist immer mit (inter-)subjektiv hervorgebrachtem (Welt-)Wissen verflochten. Zwar ist eine textlinguistische Analyse so stark mit individuellen Fachkenntnissen verbunden (objektive Denkvorgänge, eine Identität des Fachwissens bei allen Sprachteilhabern sind ein unerreichbarer Idealfall), dass eine Analyse, die alle Zweifel beseitigt und alle Fragen berührt bzw. beantwortet, prinzipiell nicht gegeben werden kann, doch sollte es eine gänzlich regelgeleitete Analyse sein, ohne einen Interpretationsspielraum zu lassen. Die Regelbefolgung gilt als Teil einer übergreifenden, den Textproduzenten (mit dem Gebot, Doppeldeutigkeiten zu vermeiden) wie Textrezipienten (so auch Textuntersuchenden) einschließenden Praxis.

\section{Literatur}

Adamzik, K. (2004). Textlinguistik. Eine einführende Darstellung. Tübingen: Niemeyer.

Beaugrande, R.-A. de \& Dressler, W.U. (1981). Einführung in die Textlinguistik. Tübingen: Niemeyer.

Biere, B.U. (1989). Verständlich-Machen. Hermeneutische Tradition - Historische Praxis - Sprachtheoretische Begründung. Tübingen: Julius Groos.

Brinker, K. (2007). Linguistische Textanalyse. Eine Einführung in Grundbegriffe und Methoden. Berlin: Erich Schmidt.

Busse, D. (1992). Textinterpretation. Sprachtheoretische Grundlagen einer explikativen Semantik. Opladen: Westdeutscher Verlag.

Canisius, P. \& Knipf, E. (1996). Textgrammatik: Ein Einführungskurs. Ein Lehr- und Arbeitsbuch für Anfänger. Budapest: Nemzeti Tankönyvkiadó.

Diekmannshenke, H. \& Klemm, M. \& Stöckl, H. (Hrsg.). (2011). Bildlinguistik. Theorien - Methoden Fallbeispiele. Berlin: Erich Schmidt.

Dijk, T.A. van (1980). Textwissenschaft. Eine interdisziplinäre Einführung. Tübingen: Niemeyer.

Dimter, M. (1981). Textklassenkonzepte heutiger Alltagssprache. Kommunikationssituation, Textfunktion und Textinhalt als Kategorien alltagssprachlicher Textklassifikation. Tübingen: Niemeyer.

DUDEN. (2011). Duden. Deutsches Universalwörterbuch. Mannheim-Leipzig-Wien-Zürich: Dudenverlag.

Große, E.U. (1976). Text und Kommunikation. Eine linguistische Einführung in die Funktionen der Texte. Stuttgart: Kohlhammer.

Große, F. (2011). Bild-Linguistik. Grundbegriffe und Methoden der linguistischen Bildanalyse in Text- und Diskursumgebungen. Frankfurt am Main u. a.: Peter Lang.

Hausenblas, K. (1977). Zu einigen Fragen der Texttheorie. In F. Daneš \& D. Viehweger (Hrsg.), Probleme der Textgrammatik II (S. 147-158). Berlin: Akademieverlag.

Heinemann, W. (1982). Textlinguistik heute - Entwicklung, Probleme, Aufgaben. Wissenschaftliche Zeitschrift der Karl-Marx-Universität Leipzig, Gesellschafts- und Sprachwissenschaftliche Reihe, 3, 210-221.

Heinemann, W. \& Viehweger, D. (1991). Textlinguistik. Eine Einführung. Tübingen: Niemeyer.

Hellwig, P. (1984). Grundzüge einer Theorie des Textzusammenhangs. In A. Rothkegel \& B. Sandig

(Hrsg.), Text - Textsorten - Semantik. Linguistische Modelle und maschinelle Verfahren (S. 51-79).

Hamburg: Helmut Buske.

Koller, W. (1975). Redensarten in Schlagzeilen. Muttersprache, 85, 400-408.

Reis, M. (1977). Präsuppositionen und Syntax. Tübingen: Niemeyer.

Vater, H. (2001). Einführung in die Textlinguistik. München: Wilhelm Fink. 